\title{
Mandibular myxoma
}

\begin{abstract}
The mandibular odontogenic myxoma is a tumor of mesenchymal origin. Represents 0.5 to 17.7 per cent of odontogenic tumors in different series and the $3^{\text {rd }}$ in frecuency. It is a histologically benign but locally aggressive and prone to tumor recurrence, which determines treatment. We report 2 cases one case is a 33 years patient and the second one is 60 years patient both diagnosed with mandibular myxoma surgically treated with segmental mandibulectomy with safety margins and immediately reconstruction with titanium plate 2.0 and 2.4 .
\end{abstract}

Keywords: odontogenic myxoma, mandibular reconstruction
Volume 6 Issue 2 - 2017

\section{Felipe Kornecki Radzinski}

Department of Dentistry, University of Eastern Republic of Uruguay, Uruguay

Correspondence: Felipe Kornecki Radzinski, Department of Dentistry, University of Eastern Republic of Uruguay, Uruguay, Email felipekornecki@gmail.com

Received: September 20, 2016 | Published: January 26, 2017

\section{Introduction}

Myxoma is a benign but locally aggressive and very relapsing tumor that affects the connective tissue. It can be located in both soft tissues and bone and although it can be found in different areas of head and neck it's most frequent presentation is in the jaws, where it is called Odontogenic Myxoma. Its most usual presentation is in the posterior mandibular sector. The most common age of presentation is the third decade of life, being less frequent in children and over $50 .{ }^{1-11}$ It is a slow-growing tumor that can reach a considerable size many times without producing any symptomatology. Some authors describe paraesthesia and pain. As its size increases it expands the cortical bone and can cause tooth dislocations, altering the occlusion. ${ }^{8}$ Embryologically derived from the mesenchymal portions of the dental germ, papilla, follicle, periodontal ligament. It is the third odontogenic tumor in frequency after Odontoma and Ameloblastoma. In its anatomy pathology presents fusiform cells in a myxoid stroma. As it is a tumor devoid of capsule, it has the potential capacity to penetrate the trabecular spaces, to cause the destruction of the cortical bone and to perforate and to invade the adjacent soft tissues. This ability to invade the bone is accompanied by a high rate of relapses. The majority of the authors agree that the correct treatment is radical resection with radiological safety margins of at least $0.5-1 \mathrm{~cm}$.

\section{Clinical cases}

\section{First clinical case}

It is a patient of 33 years, with no personal history to emphasize that he consulted his dentist in 2006 for presenting a swelling in the lower right area. The clinical and radiological diagnosis of the inferior third molar 4.8 is made retained with an associated radiolucent lesion. The dentist indicates the extraction. The patient does not know the indication (Figure 1). In 2007, a year later, he visits his dentist again, and the latter requests a new orthopantomography, where an increase in size of the radiolucent area is observed distal to the third molar. The increase in size of the radiolucent area is from distal to the $3^{\text {rd }}$ to the base of the mandibular condyle and the coronoid process. There is a need for a biopsy (Figure 2). A biopsy is performed in 2007 and the pathological anatomy confirms the diagnosis of Mandibular Odontogenic Myxoma. Routine preoperative examinations are requested and a CT scan is ordered (Figure 3).

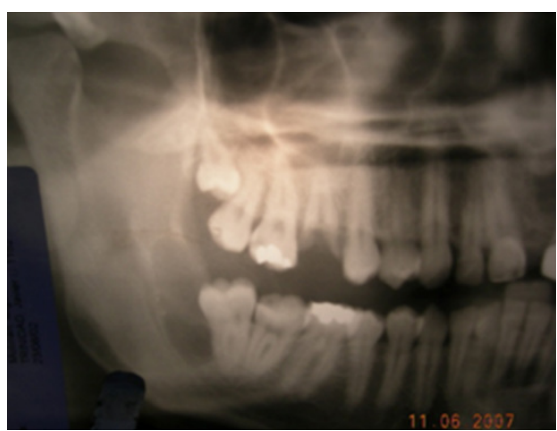

Figure I Radiolucent lesion associated with right 3rd molar.

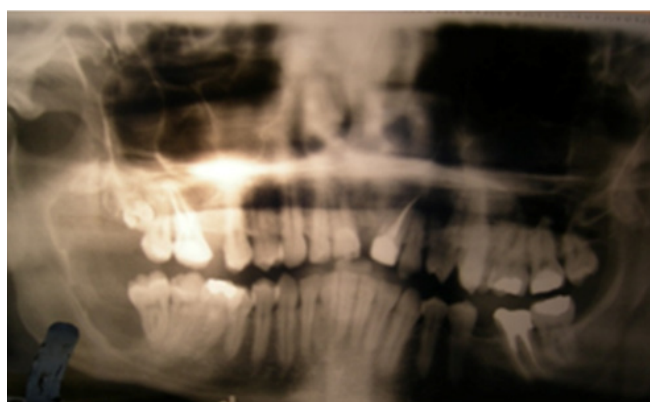

Figure 2 Radiolucent area associated to the 3 rd molar increased in size.
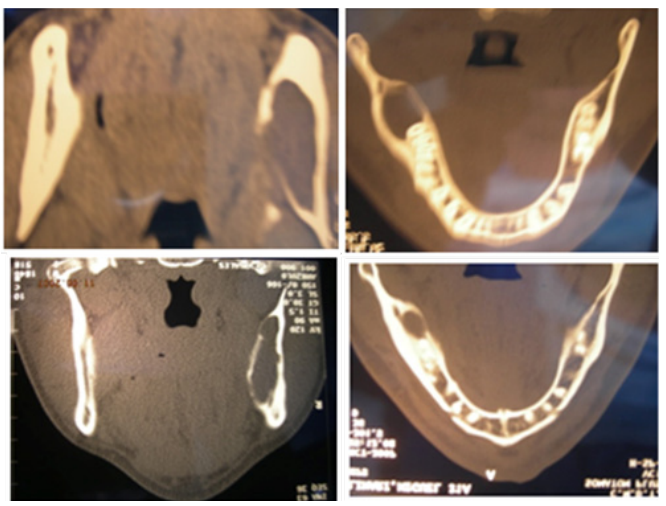

Figure 3 Computed tomography, frontal and horizontal cut. 


\section{Treatment plan}

$1^{\text {st }}$ Surgery: Under general anesthesia and nasotracheal intubation, the internal field is performed and a pharyngeal packing is placed. Intermaxillary fixation is performed with intermaxillary fixation screws and $0.5 \mathrm{~mm}$ wire.

The infiltration of the region with Mepivacaine at 2: Surgery is begun with an intraoral approach, with an incision that circumscribes 4.6 and 4.7 teeth per vestibular and lingual and ascends the anterior edge of the branch, taking into account the planned margins. Subperiosteal take-off is performed at level 44 and a safety margin of $1 \mathrm{~cm}$ above the tumor is sought in the branch. By the intraoral approach the osteotomy with strawberry number 8 is started, under irrigation with physiological serum at the proximal and distal ends of the lesion. The extraoral approach is then performed in the mandibular sub-angle region and the mandible is accessed by blunt dissection, ligating the artery and facial vein, and preserving the mandibular nerve branch of the Facial. Before the end of the osteotomy the parallel reconstruction plates 2.3 are positioned and the position of the screws is defined with the screws after the mandibular resection is completed (Figure 4). The plates are removed and the osteotomy is continued until finished. The mandibular tumor is resected without disarticulation. The reconstruction is performed with 2 parallel plates 2.3 a closure is made by plans. The patient is controlled in the polyclinic of surgery and Traumatology Bucomaxilofacial of the SMI. The patient progresses favourably (Figures 5-7).

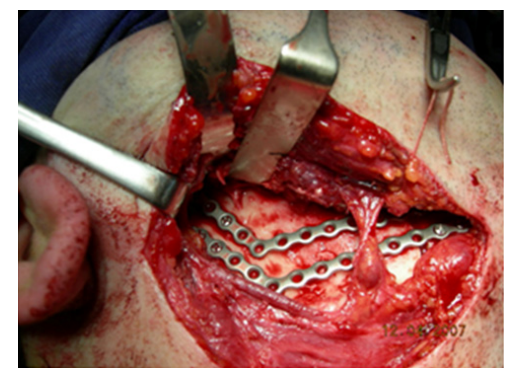

Figure 4 Parallel plates 2.3 in position, prior to mandibular resection.
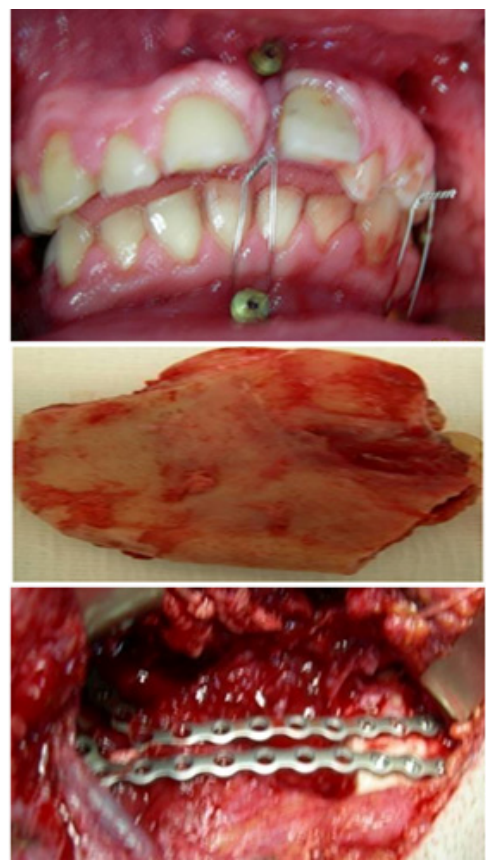

Figures 5A-5C Images of first surgery;A: Intermaxillary fixation; B: Operative part; C: Reconstruction plates in position.
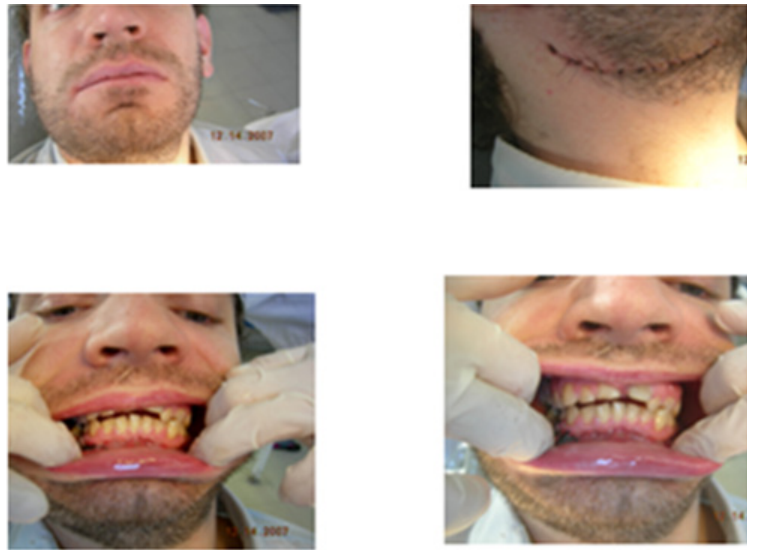

Figure 6a-6d Clinical control; 6a: patient in closed mouth occlusion; 6b: Control of the cervical approach; $6 \mathrm{c}$ and $6 \mathrm{~d}$ : control of occlusion and facial midline.
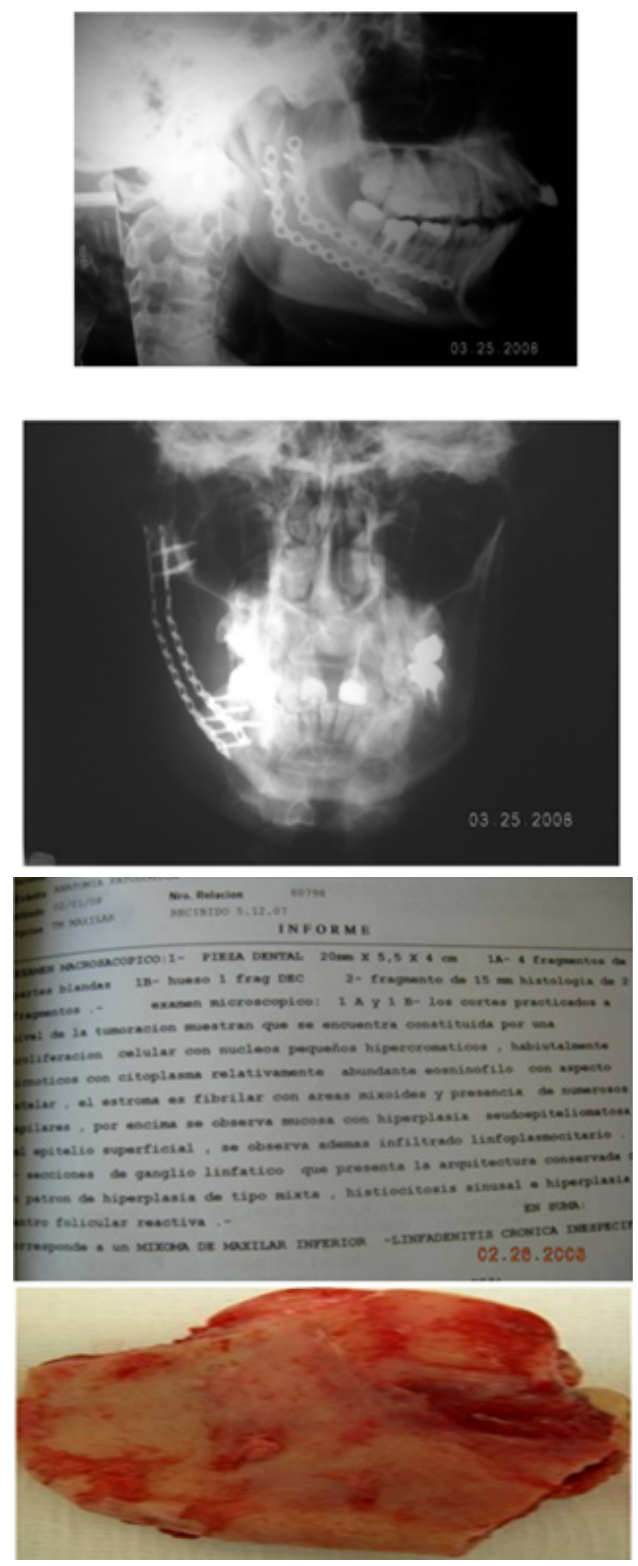

Figure 7a-7d Rx control and tomographic post operative of the first surgery. 
$2^{\text {nd }}$ surgery: Computed tomography with reconstruction is requested in the 3 planes of the space. 3D (Figures 8A-8E) (Figure 9). Due to the extension of the lesion in the mesio-distal direction, a posterior iliac crest graft is taken, as the defect is greater than $7 \mathrm{~cm}$. The iliac crest graft is taken by the Traumatologist. The graft is designed, and the traumatologist approaches it with the patient in the ventral decubitus, and once it is done, it performs the local hemostasis and closure by placing a suction drainage (Figures 10A-10D). The patient is placed in the dorsal decubitus position. The operated region is approached by the neck again, the graft is molded with strawberry under physiological serum, and it is adapted and fixed to the mandibular reconstruction plate of the first surgery (Figure 11). From a clinical and imaging point of view, the patient progresses favourably (Figures 12A \& 12B).

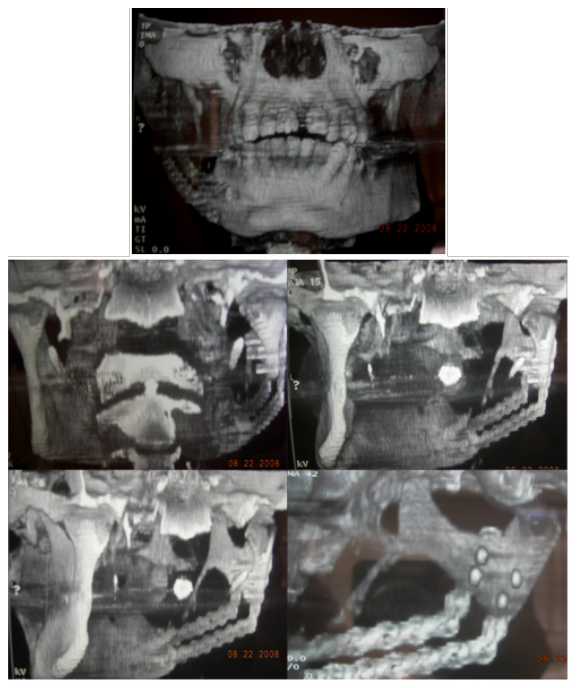

Figure $\mathbf{8 a - 8 e}$ Computed tomography with reconstruction is requested in the 3 planes of the space 3D.

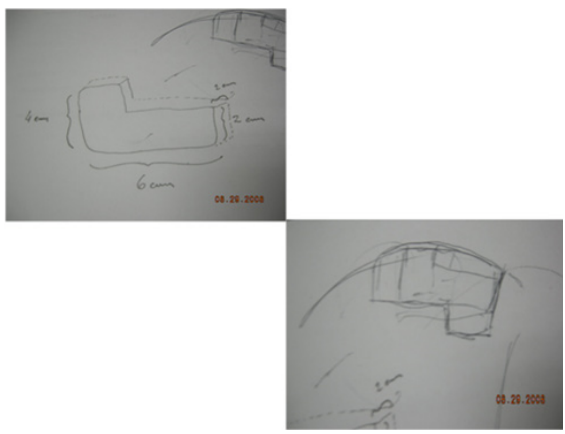

Figure 9 Graft design.

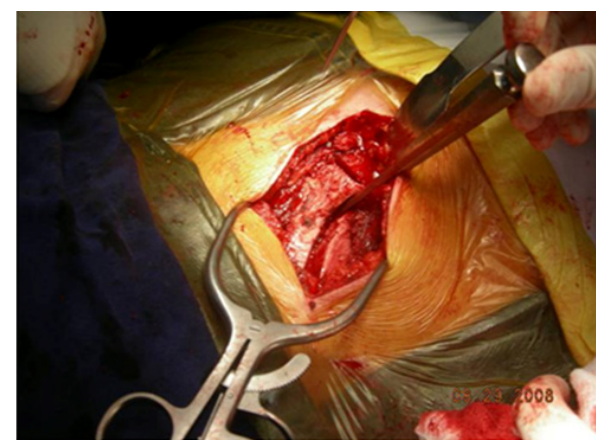

Figure 10a Implantation of posterior iliac crest graft.

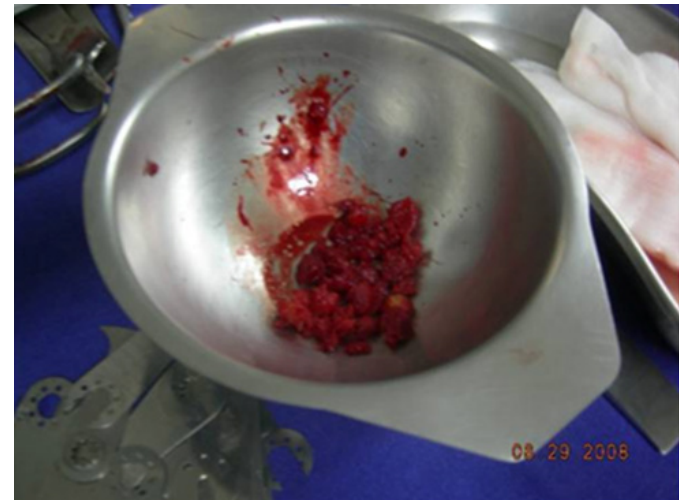

Figure I0b Particulate grafting.

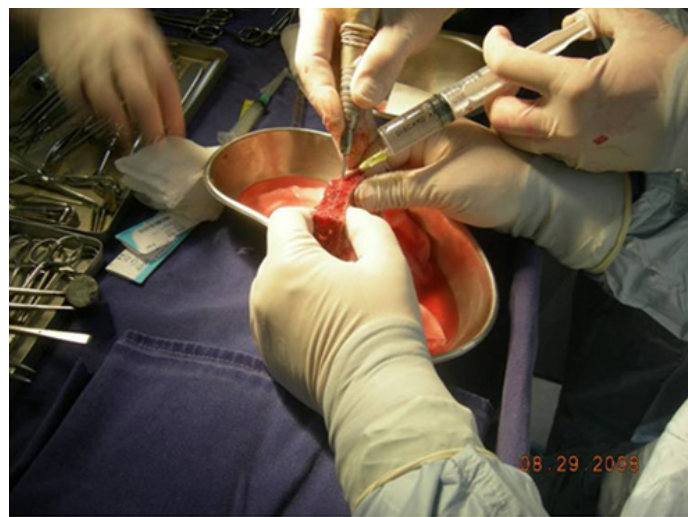

Figure 10c Carving of the graft.

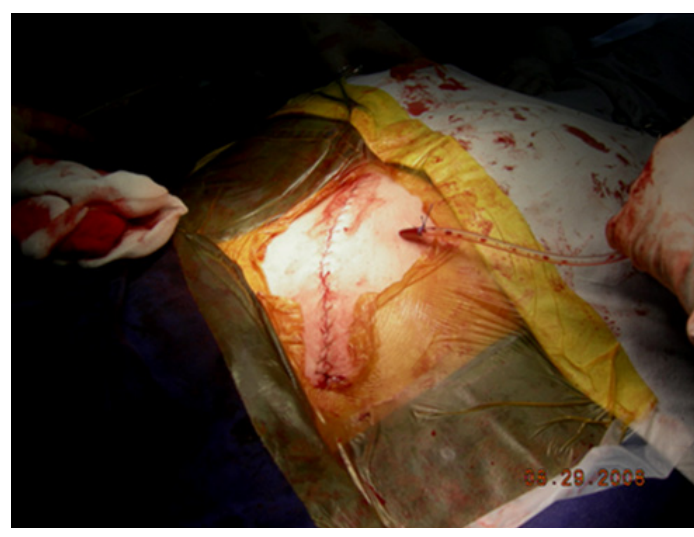

Figure I0d The posterior approach is sutured and a drain is placed.

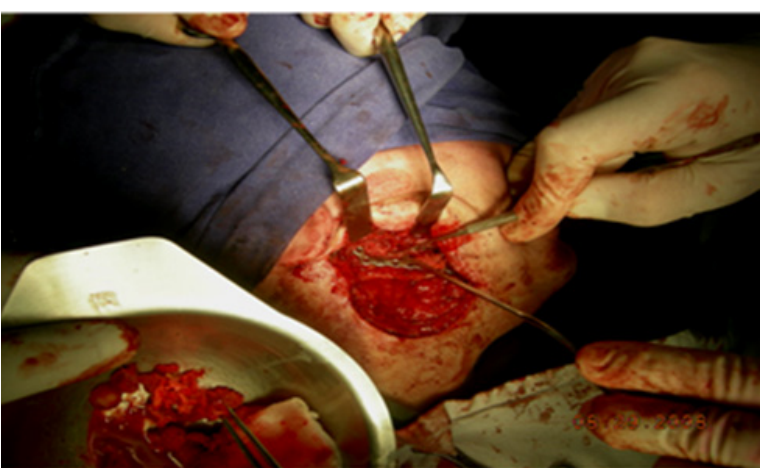

Figure II Graft in position. 

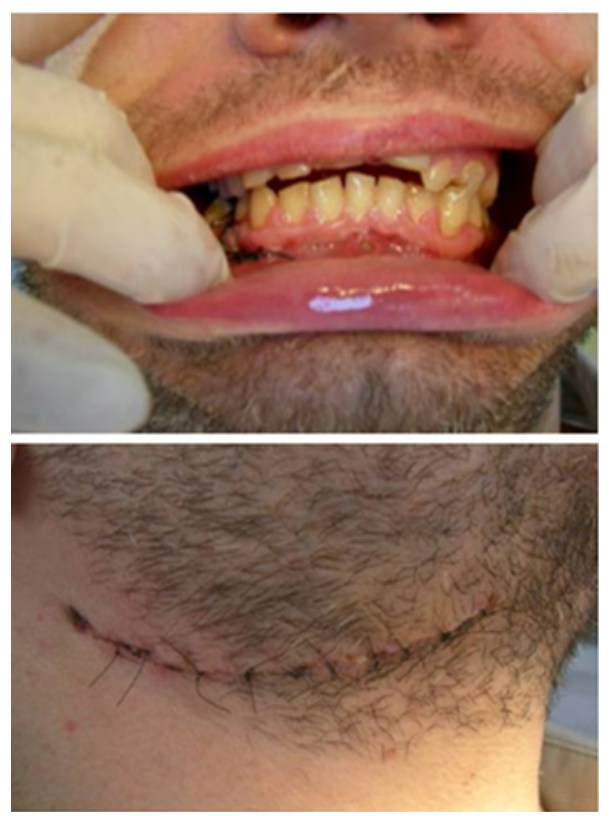

Figure I 2a, I 2b Control of occlusion and collision to the neck.

\section{2nd patient}

Man of 60 years, married with children from Artigas. Hypertensive-smoker, it presents with a swelling of the left mandibular posteroinferior area of years of evolution with diagnosis by mandibular myxoma biopsy (Figure 13). Preoperative the patient attends with Figures requested by the treating colleague. I request a conventional tomography with cuts in the three planes of the space as a complement for the surgery (Figure 14) (Figure 15). Preoperative studies are requested, consultation with an internist and anaesthesiologist. Surgical intervention is planned with general anesthesia and nasotracheal intubation.

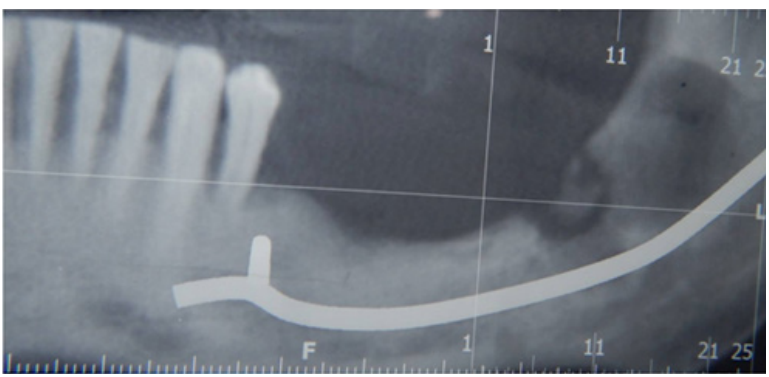

Figure 13 OPT- pre-operative.

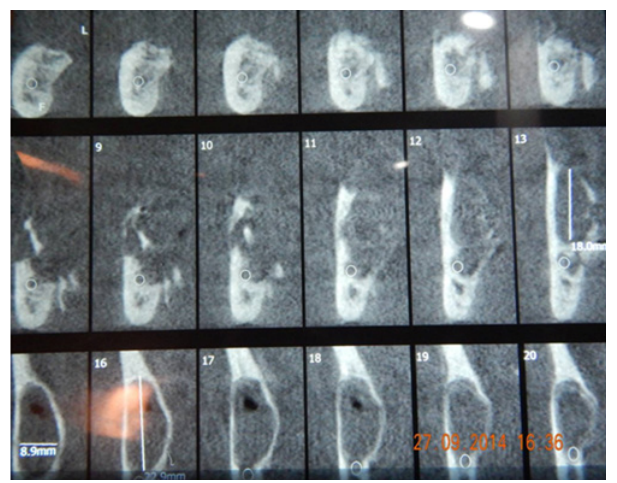

Figure I4TAC - frontal cuts.

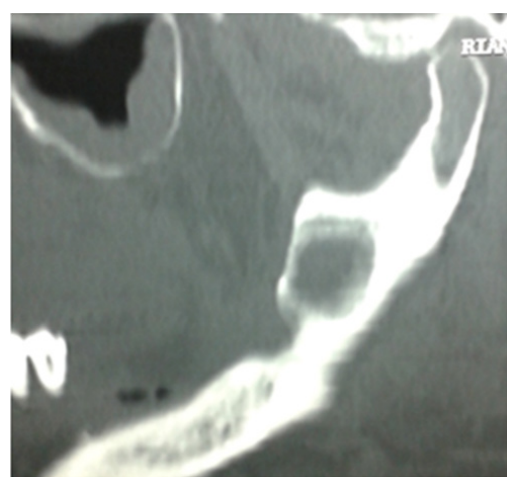

Figure 15 Sagittal cut.

\section{I st surgery}

The surgical plan according to the clinical and imaginary lesion extension consisted in-Intraoral approach of the anterior border of mandibular ascending limb. Subperiosteal dissection of the inner side of the branch. Clamping and cutting of the lower blister pack. Avulsion of the 33 the part 33 is removed as a margin of safety and is ascended by the branch to extend $1 \mathrm{~cm}$ above the lesion. Cervical approach, dissection by planes to the mandibular plane. Ligation of the facial package and preservation of the mandibular nerve. Adaptation and marking in the mandible of the position of the mandibular reconstruction plate. Segmental mandibulectomy from the alveolus of the 33 , in the center of the alveolus, to the base of the coronoid process and the mandibular condyle with oscillating saw (Figure 16) (Figure 17). Final molding and fixation of the reconstruction plate with cortico-cortical screws. The lower dental package is attached and the hemostat is removed. Closure by intraoral and extraoral plans. It is controlled with computed tomography prior to the $2^{\text {nd }}$ intervention (Figure 18) (Figure 19).

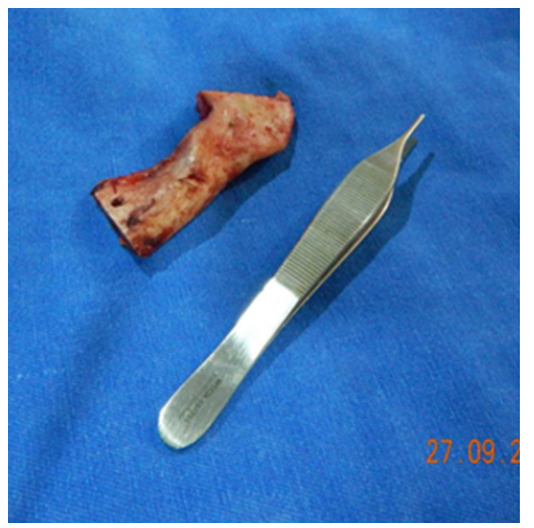

Figure 16 Operative part.

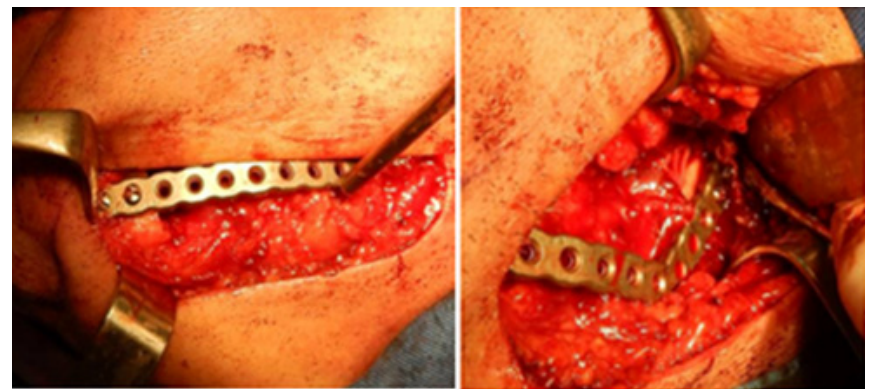

Figure 17 Cervical approach and reconstruction plaque placement 2.4. 


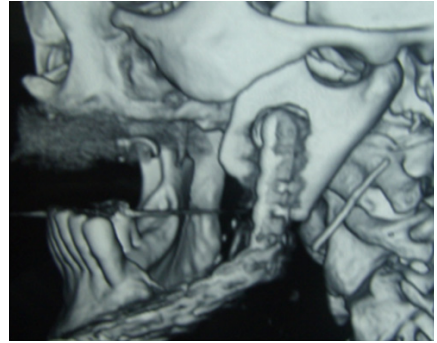

Figure 18 Tomographic control of the Ist surgery.

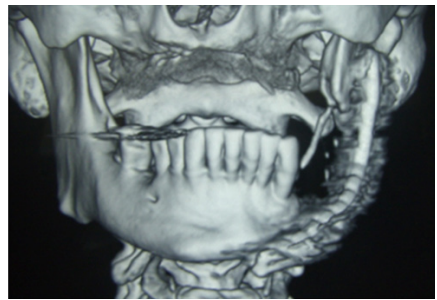

Figure 19 Misadaptation of the first reconstruction plaque observed in the frontal tomography.

\section{2nd surgery}

Under general anesthesia and nasotracheal intubation, it is planned to take an anterior iliac crest graft. The Traumatologist performs the procedure of taking the graft. At the same time we performed the approach to the neck by the anterior cervical scar. We dissect by planes until access to the plate of reconstruction of the first surgery. Placing the patient under occlusion with wire and intermaxillary fixation screws removed the old plate and adapted a new one 2.4. We work on the obtained graft, with strawberries and strawberries under irrigation with physiological serum. We adapted it to the surgical bed and fixed it to the mesial and distal ends of the resection with low profile 2.0 plates. On the graft was placed a plate 2.4 we performed the toilet of the wound and closed the wound by planes with Vicryl 3.0 and needle RV (Figures 20-23).

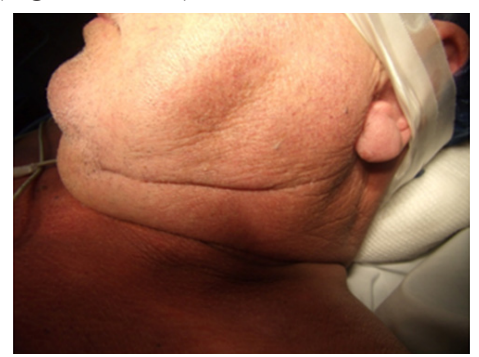

Figure 20 Scar of the first surgery.

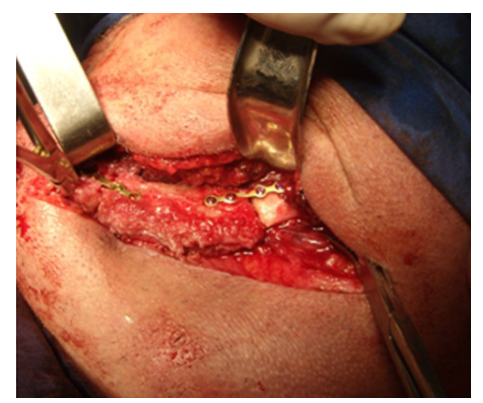

Figure 2I The first reconstruction plate was removed. The graft was adapted and fixed to the ends of the resection with mesial and distal 2.0 plates. A reconstruction plaque was attached to the graft and to the mesial and distal mandibular ends.

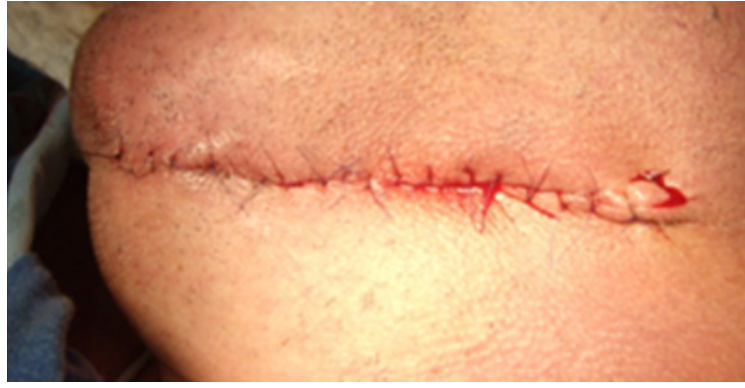

Figure 22 Close up of surface planes.
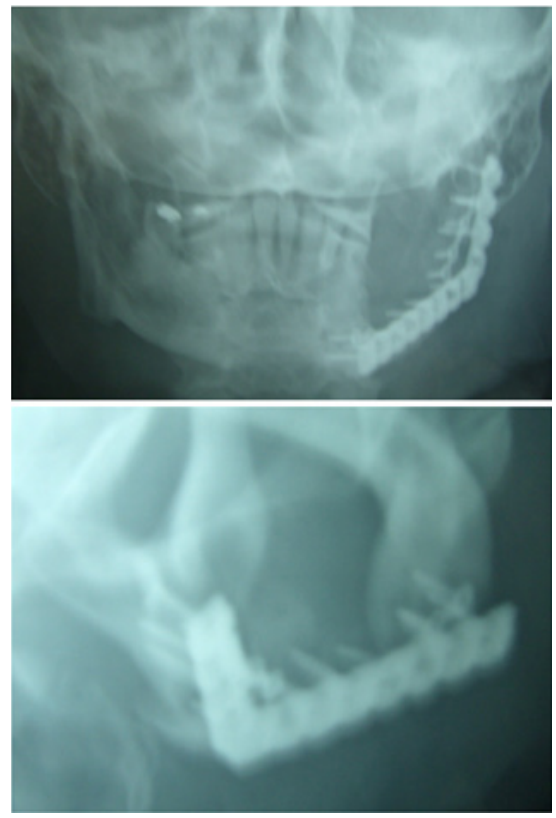

Figure 23 Frontal and lateral radiographic control postoperative.

\section{Discussion}

Odontogenic myxomas are aggressive benign tumors of infiltrating and recurrent behavior after inadequate surgical resection. Being devoid of capsule the tumor is introduced into the trabecular spaces, so it is essential to control the margin of safety. Myxoma treatment is surgical, with different protocols in the literature. The ideal treatment is based on the use of a microvascular flap.

A. Advantages of reconstruction with Microsurgery:

1. Primary reconstruction with a high percentage of success.

2. Fewer complications.

3. Flexibility of the design.

4. Increased tumor respectability.

5. Minimal aesthetic sequel and functionality in the donor zone.

B. Disadvantages of reconstruction with microsurgery:

1. Special equipment and training.

2. Need to find a recipient artery and vein.

3. High cost of intervention.

4. Duration of surgery. 


\section{Indications of microsurgery}

a. Reconstruction of soft tissues.

b. Complex facial skull resections.

c. Mandibular and maxillary reconstructions.

d. Certain cases of facial paralysis.

e. Hemifacial Microsomy.

f. Total nasal reconstruction.

The most used are the iliac crest and the fibula. In the case of using the microvascular iliac crest the dental implants can be placed in the same surgical act 2 and 3 the iliac crest gives us the width and height for immediate reconstruction. In the case of using the microvascular iliac crest the dental implants can be placed in the same surgical act 2 and 3 the iliac crest gives us the width and height for immediate reconstruction. In case of using fibula, it can be used as it is taken from the donor zone. In case of simple use the fibula, there are 2 drawbacks for immediate reconstruction does not give adequate height to be able to reconstruct immediately with implants of osseointegration. The use of fixation means in the conformation of the mandibular arch with the fibula makes difficult the posterior placement of the implants. 1 and 2 In that case it is possible to place the fibula in a double bar with the risk of compromising its vascularization or otherwise after local adaptation, perform the Osteogenic Distraction of the same 1 and 6. With my surgical team we use the protocol of Prof. A Pogrel it consists in performing the segmental resection of the pathologically compromised segment and the immediate reconstruction with a reconstruction plate performing an intraoral and extraoral approach.

\section{Description of protocol}

a. Autogenous graft secondary to the first surgery, in 6 or 8 weeks with an extraoral approach.

b. Removal of the Reconstruction Plate 4 or 6 months after the autogenous graft is placed if possible.

c. Skin graft and vestibuloplasty 6 or 8 weeks after removal of the Reconstruction Plate.

d. Osseointegration implants 8 to 12 weeks after vestibuloplasty.

e. $2^{\text {nd }}$ Implant stage 4 to 6 months after implantation.

f. Prosthetic Reconstruction.

\section{Conclusion}

We presented two male patients, one of 33 patients and another of 60 years who presented the same pathology in the mandibular postero-inferior sector, odontogenic Mixoma, who underwent a radical surgical treatment of mandibulectomy without disarticulation and reconstructed with titanium plate of mandibular reconstruction $2.3 \mathrm{~mm}$ and $2.4 \mathrm{~mm}$. In a $2^{\text {nd }}$ Time, 6 months later the patients are operated and one of them is placed a posterior iliac crest graft, the first patient and the other anterior iliac crest. The evolution of both has been very good clinical and imaging. The first patient is in condition to be rehabilitated and has not attended to its realization. The $2^{\text {nd }}$ patient is awaiting the evolution of the graft.

\section{Acknowledgments}

None.

\section{Funding}

None.

\section{Conflicts of interest}

The authors declare there is no conflict of interests.

\section{References}

1. Clinical protocols of the spanish society of oral and maxillofacial surgery Spanish Society of Oral and Maxillofacial Surgery; 2006.

2. Carlos Navarro Cuellar Jose Luis Cebrian Carter, Alvaro Garcia Rozado Gonzalez Coordinators-Fernando Garcia Marin. Alvaro Garcia - Rosado Gonzalez, Florencio Monge Gil.

3. Alvimar Lima, Claudia MCK, Renata C, et al. Odontogenic Myxoma in Mandibula. Aracatuba Dental Journal. 2003;24(2):23-27.

4. Zhao Gong, Zhang S, Ren ZH, et al. Application of American association of oral and maxillofacial surgeons anteo-medial thigh flap for the reconstruction of oral and maxillofacial defects. J Oral Maxillofac Surg. 2014;72(6):1212-1225.

5. Zhao L, Shang H, Chen X, et al. Biomechanical Analysis of a Cuvilinear Distractor Device for Correcting Mandibular Symphyseal Defects. J Oral Maxillofac Surg. 2014;72(6):1158-1167.

6. Emeka N Kenke, Agaimy A, Cornelius VW, et al. Mandibular Reconstruction Using Intraoral Microvascular Anastomosis Following Removal of an Ameloblastoma. J Oral Maxillofac surg. 2013;71(11):1983-1992.

7. Katinska K, Philipp J, Zdzislav K, et al. Odontogénic Mixoma. Diagnostic and therapeutic challenges in paediatric and adults patients-A case series and review of the literature. Journal of Craneo Maxillo-facial Surgery. 2011;40(3):271-276.

8. Abdenour S, Benhalima H. Benign odontogenic tumors-epidemiological analysis of 97 cases In the Algerian population. Rev Stomatol Chir Maxillofac Chir Orale. 2013;114(2):67-71.

9. Parr JM. Flow-Through Flap for Salvage of Fibula Osseocutaneous Vascular Variations. Surgical Approach And Proposed Modification of Its Classification. J Oral and Maxillofac Surg. 2013;72(6):1197-1202.

10. James R Hupp, Edward Ellis, Myron RT. Contemporary oral and maxillofacial surgery. 6th ed. Spain: Elsevier Mosby; 2014:1-718.

11. Carlos Navarro Vila. Tratado de cirugia oral y maxilofacial. Tomo III. Generalidades de microcirugía. Capitulo. 2nd ed. Aran, Madrid, España; 2009 